\title{
The Use of Lithium Carbonate in the Preparation for Definitive Therapy in Hyperthyroid Patients
}

\author{
Fulya Akin Guzin Fidan Yaylali Mehmet Bastemir \\ Department of Endocrinology and Metabolic Diseases, School of Medicine, Pamukkale University, Denizli, Turkey
}

\section{Key Words}

Thyrotoxicosis - Lithium carbonate therapy $\cdot$ Preoperative preparation - Radioiodine therapy

\begin{abstract}
Objective: The aim of this study was to elucidate the effectiveness of lithium carbonate prior to thyroidectomy or radioiodide therapy in patients with thyrotoxicosis. Subjects and Methods: Lithium carbonate was used as preoperative preparation or radioiodide therapy in 5 patients with Graves' disease and in 1 patient with toxic multinodular goiter because of side effects of thionamide in 5 patients and ineffectiveness of antithyroid medication in the remaining patient. Results: All 6 patients had a benign course following treatment without thyroid storm. No adverse effects or complications of lithium carbonate were observed. Conclusion: This report shows that lithium carbonate can be safely used preoperatively or prior to radioiodide therapy in circumstances where antithyroid medications are contraindicated and are ineffective in obtaining an euthyroid status.
\end{abstract}

Copyright @ 2008 S. Karger AG, Basel
(C) 2008 S. Karger AG, Basel

1011-7571/08/0172-0167\$24.50/0

Fax +4161306 1234

E-Mail karger@karger.ch

www.karger.com
Accessible online at: www.karger.com/mpp

\section{Introduction}

Lithium carbonate is a medication widely used to treat bipolar affective disorders and acute manic depressive disorders. It has also been shown that lithium carbonate has antithyroid actions similar to those of inorganic iodide and has proven effective in doses of $300 \mathrm{mg} \mathrm{3-4}$ times daily [1]. It is used primarily to provide temporary control of thyrotoxicosis in patients who cannot take thionamide drugs and/or iodide. When administered (800-1,200 mg daily) to patients suffering from Graves' thyrotoxicosis, the serum $\mathrm{T}_{4}$ and $\mathrm{T}_{3}$ levels have been shown to decrease by as much as $35 \%$ and most patients become clinically euthyroid within 2 weeks of treatment. In a previous in vitro study, it was shown that lithium carbonate inhibited colloidal droplet formation of $3^{\prime}$ and TSH-stimulated ${ }^{131}$ I release from thyroid cells. Therefore, lithium carbonate is considered as a possible treatment for thyrotoxicosis as a 'second-line' drug [2]. In patients with large goiters, but contraindications to thyroidectomy (i.e. cardiac disorders), lithium might represent a useful adjunct to radioiodide to achieve, more rapidly, control of hyperthyroidism [3].

In these reports, we present 5 patients with Graves' disease and 1 patient with toxic multinodular goiter treat- 
ed with lithium carbonate preoperatively or prior to radioiodide therapy. Lithium treatment was selected because of complications from thionamide medications in 5 patients and inadequate control by thionamide in 1 .

\section{Case Reports}

Case 1

A 35-year-old woman presented with symmetrical, tender and palpable purpuric lesions on her lower legs. She had been treated with propylthiouracil (PTU) for Graves' disease for 6 years. Furthermore, she suffered from Marfan's syndrome with severe mitral regurgitation, tricuspid regurgitation and rapid atrial fibrillation, which were aggravated by hyperdynamic circulatory conditions associated with coexistent hyperthyroidism and Graves' disease. Her mother also had Marfan's syndrome.

The patient had been treated with digoxin for heart failure for 4 years. She developed an erythematous rash after receiving PTU for a few days. The patient was diagnosed as having PTU-induced antineutrophil cytoplasmic antibody (ANCA-MPO) positive vasculitis with markedly elevated ANCA anti-MPO antibodies. Her PTU therapy was discontinued, and she was treated with prednisone and cyclophosphamide. She was also given propranolol, coumadine and antibiotics. The patient became euthyroid after treatment with lithium carbonate and radioactive iodide. Her skin lesions improved and her ANCA titers decreased to $41 \mathrm{U} / \mathrm{ml}$ after 6 weeks of treatment.

\section{Case 2}

A 63 -year-old woman was diagnosed as having toxic multinodular goiter. Due to elevated liver enzymes [ $\geq 3$ times elevation of the upper limit of normal of liver function tests; AST: 112 IU/1 (13-35), ALT: 168 IU/1 (10-35)] lithium carbonate therapy was started rather than PTU or methimazole. Further laboratory examination showed positive antimitochondrial antibodies and liver biopsy was consistent with primary biliary cirrhosis. Radioactive iodine (RAI) therapy was the selected treatment. Prior to RAI she was placed on $300 \mathrm{mg}$ of lithium 3 times a day for 2 weeks. She was euthyroid 2 weeks after starting lithium. After treatment with ${ }^{131} \mathrm{I}$, she became euthyroid.

\section{Case 3}

A 62-year-old woman diagnosed as having Graves' disease and nodular goiter was scheduled for thyroidectomy. She was initially treated with PTU $4 \times 200 \mathrm{mg}$ and propranolol $3 \times 40 \mathrm{mg}$ but remained hyperthyroid. She therefore received dexamethasone ( $1 \mathrm{mg} /$ day) and lithium carbonate (300 $\mathrm{mg}$ t.i.d.). After 2 weeks of combination therapy she was euthyroid and was able to be operated on.

\section{Case 4}

A 22-year-old woman was diagnosed as having Graves' disease and hyperthyroidism while being evaluated for bilateral popliteal deep venous thrombosis. She was treated with coumadine $(1 \times$ $5 \mathrm{mg})$ and methimazole $(3 \times 10 \mathrm{mg})$. As the therapeutic international normalized ratio level had not been obtained and drugdrug interaction was suspected, her methimazole was discontin-
Table 1. Thyroid function tests of patients before and after lithium carbonate therapy

\begin{tabular}{|c|c|c|c|c|c|c|}
\hline \multirow[t]{2}{*}{ Cases } & \multicolumn{3}{|c|}{ Before lithium therapy } & \multicolumn{3}{|c|}{ After lithium therapy } \\
\hline & $\begin{array}{l}\mathrm{FT}_{3} \\
\mathrm{pg} / \mathrm{ml}\end{array}$ & $\begin{array}{l}\mathrm{FT}_{4} \\
\mathrm{ng} / \mathrm{dl}\end{array}$ & $\begin{array}{l}\text { TSH } \\
\text { IU/ml }\end{array}$ & $\begin{array}{l}\mathrm{FT}_{3} \\
\mathrm{pg} / \mathrm{ml}\end{array}$ & $\begin{array}{l}\mathrm{FT}_{4} \\
\mathrm{ng} / \mathrm{dl}\end{array}$ & $\begin{array}{l}\text { TSH } \\
\mathrm{IU} / \mathrm{ml}\end{array}$ \\
\hline Case 1 & 15.39 & 1.96 & 0.010 & 3.10 & 1.14 & 0.005 \\
\hline Case 2 & 6.88 & 1.87 & 0.005 & 3.72 & 1.25 & 0.005 \\
\hline Case 3 & 22.2 & 7.77 & 0.007 & 4.09 & 2.04 & 0.007 \\
\hline Case 4 & 2.44 & 0.6 & 0.005 & 2.01 & 0.431 & 0.819 \\
\hline Case 5 & 9.51 & 1.83 & 0.005 & 3.49 & 0.579 & 0.011 \\
\hline Case 6 & 5.73 & 2.79 & 0.007 & 2.24 & 2.87 & 0.011 \\
\hline
\end{tabular}

$\mathrm{FT}_{3}=$ Free triiodothyronine; $\mathrm{FT}_{4}=$ free thyroxine; $\mathrm{TSH}=$ thyroid-stimulating hormone.

ued $[3,4]$. Thereafter, lithium carbonate therapy was initiated. After 3 weeks, the euthyroid state was achieved and a therapeutic international normalized ratio level was also obtained. She was managed with RAI therapy.

\section{Case 5}

A 40-year-old man had been receiving thyramazole 3 times daily (t.i.d.) for Graves' disease. Because of elevated liver enzymes [ $\geq 3$ times elevation of the upper limit of normal of liver function tests; AST: 102 IU/1 (13-35), ALT: 112 (10-35)], the dose of thyramazole could not be increased and he remained hyperthyroid. Later, thyramazole was discontinued and lithium carbonate 300 mg t.i.d. was started; he became euthyroid with normal blood pressure and pulse rate in 3 weeks and was referred for thyroidectomy.

\section{Case 6}

A 37-year-old woman was treated with lithium carbonate 300 mg t.i.d for Graves' disease because she had elevated liver enzymes $[\geq 3$ times elevation of the upper limit of normal of liver function tests; AST: 59 IU/1 (13-35), ALT: 188 IU/1 (10-35)]. She was clinically euthyroid with normal blood pressure and pulse rate in 2 weeks and referred for thyroidectomy.

Cases 5 and 6 were given lithium carbonate therapy prior to thyroidectomy because of concern for using antithyroid medications in patients with hepatic dysfunction. The thyroid function tests of the patients before and after lithium carbonate therapy are shown in table 1 . All the patients had a successful outcome or postradiation ablation without postoperative thyroid storm or surgical recurrence.

\section{Discussion}

Among the side effects of lithium carbonate, goiter development and hypothyroidism are well known [4]. The other effects on the thyroid include decreased iodide re- 
lease, impaired hormone synthesis and probably decreased $\mathrm{T}_{4}$ deiodination.

The indications for the use of lithium carbonate have not been properly established, probably because of the effectiveness of regular antithyroid drugs and the fear of possible complications of lithium carbonate treatment, including central nervous system disturbance (confusion, blurring of vision, clumsiness, seizures and coma), arrhythmias (ventricular irritability, sinus node dysfunction and sinoatrial block), hyperparathyroidism and calcium level changes, weight gain and nephrogenic diabetes insipidus. However, serious complications rarely occur unless the lithium carbonate serum levels exceed 1.4 $\mathrm{mEq} / \mathrm{l}$ [3]. One patient with severe thyrotoxicosis was treated with lithium carbonate prior to thyroidectomy. The intraoperative findings were unremarkable, with normal blood pressure and pulse rate and minimal blood loss. The postoperative course was uneventful. This case demonstrates the effectiveness of lithium carbonate in thyrotoxic emergencies in which a rapid and safe inhibition of thyroid hormone secretion occurred [5].

Radioiodide therapy is a well-established treatment for Graves' hyperthyroidism. Several factors affect its efficacy, including the short persistence of radioiodide in the thyroid gland. In hyperthyroid Graves' patients, radioactive iodide uptake is enhanced by TSH receptor antibody [6]; however, radioiodide is also rapidly discharged because of its increased turnover. The effectiveness of radioiodide therapy may be reduced by pretreatment with PTU but not with methimazole [7]. Lithium carbonate can significantly affect the kinetics of iodide by reducing its release from the thyroid gland, thus increasing its retention. However, lithium carbonate is rarely used to potentiate the therapeutic effect of radioiodide therapy. Beneficial effects of lithium carbonate in combination with radioiodide have been reported in cases of thyroid carcinoma [8] and in a nonrandomized study of Graves' disease [9]. However, lithium carbonate is not recommended for general use in Graves' disease but only in the patients with very short effective half-life in whom the required thyroid dose may occasionally not be achieved because too much activity would otherwise be needed [10].

A short course of lithium carbonate therapy can be considered a useful adjunct to RAI therapy for obtaining a more rapid control of thyrotoxicosis and avoiding its transient exacerbation because of methimazole withdrawal prior to RAI administration [11] or in patients who cannot tolerate or do not respond to thionamides [12]. In these reports $[11,12]$ as in our 3 cases, the patients did not show any abnormality in thyroid function tests or any other complications.

In patients with large goiters but contraindications to thyroidectomy (i.e. cardiac disorders), as in case 1 of the present study, lithium carbonate treatment possibly represents a useful adjunct to radioiodide to achieve a more rapid control of hyperthyroidism. In this regard, an additional important effect of the addition of lithium carbonate was the lack of serum free thyroxine and free triiodothyronine surge which was observed shortly after radioiodide therapy. This effect might be related to radioiodide-induced destruction, as suggested by the concomitant rise in serum thyroglobulin concentrations, or to the prevention of thyroid hormone surge after antithyroid drug withdrawal. This effect might be particularly beneficial in older patients with an underlying cardiac disease. Although none of our patients suffered from any eye complications, we believe that the combined treatment with radioiodide plus lithium carbonate might also be favorable for Graves' ophthalmopathy because of the lower risk of recurrence of hyperthyroidism, which is known to adversely affect the course of eye diseases.

\section{Conclusion}

These cases show that lithium carbonate can be safely used preoperatively or prior to RAI therapy in circumstances in which antithyroid medications cannot be used due to side effects or when they are ineffective in obtaining the euthyroid state.

\section{References}

Lithium Carbonate Therapy in

Thyrotoxicosis
1 Kristensen O, Andersen HH, Pallisgaard G: Lithium carbonate in the treatment of thyrotoxicosis: a controlled trial. Lancet 1976;1: 603-605.

2 Lesar TS, Tollefson G: Lithium therapy. Postgrad Med 1984;75:269-285.

-3 Tsunoda T, Mochinaga N, Eto T, Yamaguchi M, Tsuchiya R, Izumi M: Lithium carbonate in the preoperative preparation of Graves' disease. Jpn J Surg 1991;21:292-296.
4 Lazarus JH, Addison GM, Richards AR, Owen GM: Treatment of thyrotoxicosis with lithium carbonate. Lancet 1974;2:11601162

5 Kauschansky A, Genel M: Preoperative treatment of intractable hyperthyroidism with acute lithium administration. Eur J Pediatr Surg 1996;6:301-302. 
-6 Marcocci C, Valente WA, Pinchera A, Aloj SM, Kohn LD, Grollman EF: Graves' IgG stimulation of iodide uptake in FRTL-5 rat thyroid cells: a clinical assay complementing FRTL-5 assays measuring adenylate cyclase and growth stimulating antibodies in autoimmune thyroid disease. J Endocrinol Invest 1983;6:463-471.

7 Imseis RE, Vanmiddlesworth L, Massie JD, Bush AJ, Van Middlesworth NR: Pretreatment with propylthiouracil but not methimazole reduces the therapeutic efficacy of iodine-131 in hyperthyroidism. J Clin Endocrinol Metab 1998;83:685-687.
8 Briere J, Pousset G, Darsy P, Guinet P: The advantage of lithium in association with ${ }^{131} \mathrm{I}$ in the treatment of functioning metastasis of the thyroid cancer. Ann Endocrinol 1974;35: 281-282.

$\checkmark 9$ Temple R, Berman M, Carlson HE, Robbins J, Wolff J: The use of lithium in Graves' disease. Mayo Clin Proc 1972;47:872878.

10 Dunkelmann S, Kunstner H, Nabavi E, Eberlein U, Groth P, Schumichen C: Lithium as an adjunct to radioiodine therapy in Graves' disease for prolonging the intrathyroidal effective half-life of radioiodine. Useful or not? Nuklearmedizin 2006;45:213218.
1 Bogazzi F, Bartalena L, Campomori A, Brogioni S, Traino C, De Martino F, Rossi G, Lippi F, Pinchera A, Martino E: Treatment with lithium prevents serum thyroid hormone increase after thionamide withdrawal and radioiodine therapy in patients with Graves' disease. J Clin Endocrinol Metab 2002;87:4490-4495.

12 Ng YW, Tiu SC, Choi KL, Chan FK, Choi $\mathrm{CH}$, Kong PS, Ng CM, Shek CC: Use of lithium in the treatment of thyrotoxicosis. Hong Kong Med J 2006;12:254-259. 